INPLASY

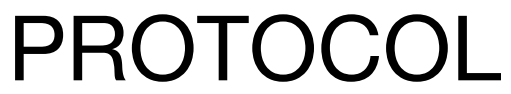

To cite: Colonetti et al. Effects of gluten free diet in patients with diabetes mellitus type1: systematic review and metaanalysis. Inplasy protocol 202060010. doi:

10.37766/inplasy2020.6.0010

Received: 02 June 2020

Published: 02 June 2020

Corresponding author: Tamy Colonetti

tamycolonetti@hotmail.com

Author Affiliation:

Universidade do Extremo Sul

Catarinense- UNESC

Support: No.

Review Stage at time of this submission: Piloting of the study selection process.

Conflicts of interest: No.

\section{Effects of gluten free diet in patients with diabetes mellitus type1: systematic review and meta-analysis}

Colonetti, T1; Mariot, $\mathrm{M}^{2}$. Colonetti $\mathrm{L}^{3}$, Costa, $\mathrm{M}^{4}$.

Review question / Objective: Gluten-free diet improves glycemic control in patients with type I diabetes mellitus? Condition being studied: According to the Ministry of Health (2019), currently around 6,000 to 8,000 autoimmune diseases are identified on average worldwide. Where the triggering of the autoimmune disease trigger is extremely linked to the consumption of gluten by individuals who have the sensitivity to develop such pathologies in the body (VOLTA et.al., 2013). Association between gluten consumption and the development of autoimmune diseases, with emphasis on type 1 diabetes mellitus, may be related to a common genetic susceptibility and to an increase in the permeability of the intestinal mucosa to antigens that trigger autoimmune mechanisms, such as gluten, in celiac disease and insulin in cases of type 1 Diabetes Mellitus (SAMÕES, 2011). However, the population that has such a pathology needs nutritional care.

INPLASY registration number: This protocol was registered with the International Platform of Registered Systematic Review and Meta-Analysis Protocols (INPLASY) on 02 June 2020 and was last updated on 02 June 2020 (registration number INPLASY202060010).

\section{INTRODUCTION}

Review question / Objective: Gluten-free diet improves glycemic control in patients with type I diabetes mellitus?
Condition being studied: According to the Ministry of Health (2019), currently around 6,000 to 8,000 autoimmune diseases are identified on average worldwide. Where the triggering of the autoimmune disease trigger is extremely linked to the 
consumption of gluten by individuals who have the sensitivity to develop such pathologies in the body (VOLTA et.al., 2013). Association between gluten consumption and the development of autoimmune diseases, with emphasis on type 1 diabetes mellitus, may be related to a common genetic susceptibility and to an increase in the permeability of the intestinal mucosa to antigens that trigger autoimmune mechanisms, such as gluten, in celiac disease and insulin in cases of type 1 Diabetes Mellitus (SAMÕES, 2011). However, the population that has such a pathology needs nutritional care.

\section{METHODS}

Participant or population: Patients with Diabetes Mellitus I.

Intervention: Gluten Free Diet.

Comparator: Patients with type I diabetes mellitus without dietary intervention.

Study designs to be included: Randomized clinical trial and case-control.

Eligibility criteria: Participants with type 1 diabetes mellitus submitted to a gluten-free diet compared to patients with AMD, but without changing the diet.

Information sources: A search strategy was developed using the following terms: "Gluten" OR "Secalin OR OR Hordein", "Gluten-Free Diet" and "Diabetes Mellitus, Type I", as keywords that were consulted in the Medical Subject Headings (ie MeSH), to search for possible synonyms A sensitive filter was created by combining these different synonyms to identify studies using the Boolean operators "OR" and "AND". Subsequently, these were searched in databases, such as: MEDLINE, EMBASE, Cochrane Library, IBECS, LILACS, and also in the gray literature (Google Scholar), for studies published until May 2020. The research was limited to studies in humans and had no language restrictions. The reference lists of all available primary studies have been revised to identify possible additional relevant citations.
Main outcome(s): Glycemic control.

Quality assessment / Risk of bias analysis: All included studies were evaluated for their methodological quality. For RCT this assessment involve the risk of bias in patient selection, blinding of participants and the researcher, incomplete result data, publication bias and other biases. The risk of bias will be classified as low risk, unclear risk and high risk, according to the Cochrane manual. For case-control studies we will used newcastle - ottawa quality assessment scale case-control studies.

Strategy of data synthesis: Two reviewers (M.L.M and M.C) selected the abstracts, as shown in the study selection criteria. The screening process was conducted at Rayyan (rayyan.qcri.org). Two investigators independently extracted data from the primary studies included in the study. Any disagreements about the inclusion or exclusion of studies were resolved by consensus. There was no consensus as a third reviewer (TC) selected the conflicting articles. The data extraction form was composed by author, year, country, patient characteristics, methods, intervention information and results of each included study. The results will be expressed using tables and graphs. Quantitative variables were presented as mean and standard deviation. Forest graphs were made to indicate the effects of the interventions for certain outcomes. For continuous results, we use mean deviation with $95 \%$ confidence intervals using RevMan software (version 5.3). The heterogeneity of the study was determined using $\left.\right|^{2}$ statistics (in which numbers greater than $75 \%$ suggest considerable heterogeneity) (DerSimonian \& Laird 1986) and p-values from the $x 2$ test.

Subgroup analysis: None.

Sensibility analysis: If necessary, sensitivity analysis using standards will be performed.

Country(ies) involved: Brazil.

Keywords: Gluten-Free Diet, Diabetes Mellitus, Type I, Systematic Review. 
Contributions of each author:

Author 1 - Tamy Colonetti - Project writing, search strategy, selection of studies, analysis of results.

Author 2 - Micheli Mariot - Selection of studies and data extraction, writing of the project.

Author 3 - Laura Colonetti - Selection of studies and data extraction.

Author 4 - Marina Costa - Selection of studies and data extraction. 